\title{
Atom capture by nanotube and scaling anomaly
}

\author{
Pulak Ranjan Gir:* \\ Theory Division, Saha Institute of Nuclear Physics, 1/AF Bidhannagar, Calcutta 700064, India
}

(Dated: November 6, 2018)

\begin{abstract}
The existence of bound state of the polarizable neutral atom in the inverse square potential created by the electric field of a single walled charged carbon nanotube (SWNT) is shown to be theoretically possible. The consideration of inequivalent boundary conditions due to self-adjoint extensions lead to this nontrivial bound state solution. It is also shown that the scaling anomaly is responsible for the existence of such bound state. Binding of the polarizable atoms in the coupling constant interval $\eta^{2} \in[0,1)$ may be responsible for the smearing of the edge of steps in quantized conductance, which has not been considered so far in the literature.

PACS numbers: 03.65.Ge, 11.30.-j, 31.10.+z, 31.15.-p
\end{abstract}

Research activities in atomic and molecular physics [1, 2] is speeding up due to the advancement of laser cooling technique [3, 4, 5, 6]. Substantial amount of people are engaged in devising suitable confining mechanism [7, 8, 9, 10] for cold atoms and molecules so that it can be stored at a temperature down to nano-Kelvin. Among different schemes for trapping and storing cold atoms; magnetic trap, dipole trap, optical trap etc are important. Present day technology is well equipped to handle atoms and molecules at nano-Kelvin temperature. Study of different properties of these super cold neutral atoms or molecules in electric or magnetic fields, created by charged wire [11, 12], current carrying conductor or by some other mechanism is now feasible.

Quantized conductance(QC) [1] is one such important property, which is usually seen when atoms are moving in the neighborhood of a charged nanotube. QC is basically discrete quantum steps in the cross section for atom capture versus voltage of the thin wire. Since the steps result from the angular momentum quantization in the attractive inverse square potential experienced by the atom, it is also called "angular momentum quantum ladder". The edge of the steps are not infinitely sharp but a little bit smeared out [1]. This exotic behavior is usually attributed to the tunneling of the neutral atoms through the inverse square potential $\left(\eta^{2}-1 / 4\right) / r^{2}$ and happens near the value $\eta=0$ of the coupling constant. This conclusion is based on the usual boundary condition that the wave-function is zero at the singularity. But we need further quantum mechanical investigation, because the peculiar nature of the inverse square potential in the interval $\eta \in[0,1)$ may give rise to bound state due to a nontrivial boundary condition which is known for a long time in mathematical physics.

Quantum mechanical behavior of the inverse square potential is subtle [13] in the sense that it lies between $1 / r^{n>2}$ and $1 / r^{n<2}$. It should be noted that for $n>2$ usually there can not be any bound states and for $n<2$ the potential is capable of forming bound states. Usually

*Electronic address: pulakranjan.giri@saha.ac.in a particle moving in inverse square potential does not form bound state or more specifically the system has negative infinite ground state in the region $\eta^{2}<0$ [14, 15]. It is however possible to form a single bound state for $\eta^{2} \in[0,1)$ by considering nontrivial boundary condition at the singularity of the potential. In this context it should be noted that the smearing of the edge of steps in QC also occurs near $\eta=0$. This is a possible hint that the the effect of smearing may have its origin in the nontrivial nature of the boundary condition.

Therefore the subject of this paper is to study the behavior of the neutral atom in $\left(\eta^{2}-1 / 4\right) / r^{2}$ potential created by the charged single-walled carbon nanotube (SWNT) and investigate the possible origin for the smearing in QC. However our analysis is equally applicable to an atom or a molecule moving in a current carrying wire or in ferromagnetic wire [16], where the magnetic interaction between the atom or molecule and the wire is responsible for the $\left(\eta^{2}-1 / 4\right) / r^{2}$ potential. It should be noted that in reality the actual interaction is highly nonlinear due to multi-pole expansion of the charge distribution of the polarized atom in the SWNT field. But the dominat part of the interaction is inverse square in nature. The other interactions are short range interaction between the atom and SWNT. The inverse square interaction is therefore responsible for the description of the long distance behavior of the atom. It is known [17, 18, 19] that inverse square inreraction may form bound state. Short distance interactions can however be taken into account through the boundary conditions imposed on the Hamiltonian, keeping self-adjointness [20, 21] of the Hamiltonian intact. Our goal is to find out suitable boundary conditions for the Hamiltonian in the inverse square interaction potential by using von Neumann's theory [20, 21] of self-adjoint extensions, so that that the system remains self-adjoint. Inverse square interaction has diverse applications starting from microscopic physics to black holes [17, 18, 19, 22, 23, 24, 25, 26, 27, 28, 29]. In our present work we use this model to explain the polarizable neutral atom capture by charged nanotube(SWNT) and thereby the possible occurrence of smearing in QC. von Neumann's method of self-adjoint extensions suggest 


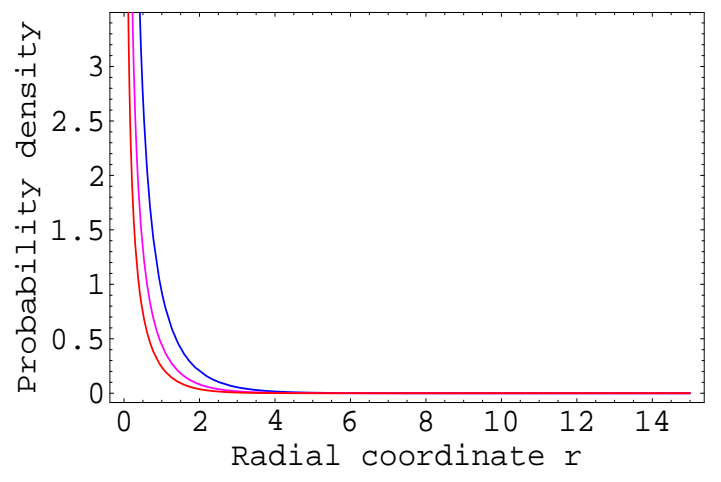

FIG. 1: (color online) A plot of the probability density of bound state as a function of the radial coordinate $r$. For blue graph $\omega=\pi / 5, \alpha=1, q=\sqrt{1 / 25}, m=1$. For pink graph $\omega=\pi / 5, \alpha=3, q=\sqrt{1 / 25}, m=1$. For orange graph $\omega=\pi / 5, \alpha=5, q=\sqrt{1 / 25}, m=1$.

the possibility of the existence of a single bound state. The coefficient of the inverse square interaction is dimensionless. The consequences of the absence of the dimensionfull coefficients in the Hamiltonian imply that there should not be any bound state in the system. But due to the quantum mechanical scaling anomaly [30, 31, 32], it can be shown that the system can form bound states. The consideration of inequivalent boundary conditions (self-adjoint extensions) is responsible for quantum mechanical anomalies [33], which in this case allows the charged nanotube (SWNT) to capture polarizable neutral atom.

We consider a polarizable neutral atom of mass $\mu$ and polarizability $\alpha$ moving in the electric field of a charged single-walled carbon nanotube with line charge $q$. The electric field $E$ of the charged nanotube induces a dipole moment $d=\alpha E$ on the neutral atom. The interaction between this induced dipole moment $d$ of the atom and the electric field of the nanotube generates a potential $V_{d}=-\frac{1}{2} \alpha E^{2}=-2 \alpha q^{2} \frac{1}{r^{2}}$. For the cylindrical symmetry of the system, it is convenient to take the $z$ axis along the nanotube. In cylindrical polar coordinates $(r, \phi, z)$, one can write the Schrödinger equation for the system as follows:

$$
\left[-\frac{\hbar^{2}}{2 \mu} \nabla^{2}-\frac{2 \alpha q^{2}}{r^{2}}\right] \Psi=\mathcal{E} \Psi
$$

where $\mathcal{E}$ is the energy of the atom in the nanotube field. We use the generic ansatz $\Psi(r, \phi, z)=\frac{1}{\sqrt{r}} R(r) e^{i m \phi} e^{i k z}$, for the wave-function to separate the Schrödinger equation (11). The radial equation after separating the angular part is

$$
H_{r} R(r) \equiv\left[-\frac{d^{2}}{d r^{2}}+\frac{1}{r^{2}}\left(\eta^{2}-\frac{1}{4}\right)\right] R(r)=\epsilon R(r) .
$$

The radial Hamiltonian $H_{r}$ has eigenvalue $\epsilon=2 \mu \mathcal{E} / \hbar^{2}$. $H_{r}$ depends on the coupling constant $\eta^{2}=m^{2}-\lambda$, where $\lambda=4 \mu \alpha q^{2} / \hbar^{2}$. It should be noted that in literature it is usually assumed that the atom does not have any bound state in the the electric field of the charged nanotube and depending on the sign of the effective coupling constant $\eta^{2}-1 / 4$ the atom either falls to the center [13] or escapes away from the nanotube towards the infinity. However as pointed out earlier we show that von Neumann's technique allows us to make the Hamiltonian self-adjoint for $-1 / 4 \leq \eta^{2}-1 / 4<3 / 4 \equiv \eta^{2} \in[0,1)$ and get a single bound state for this self-adjoint Hamiltonian.

It is also to be noted that the above interval for the coupling constant $\eta$ is actually realizable in experiment. Let us discuss it with an example. Lithium atom can be a good candidate for our example because it has been used in the experiment for cold atom [2]. For the Lithium atom with mass $\mu_{L i}=9.99 \times 10^{-27} \mathrm{Kg}$ and polarization $\alpha_{L i}=24 \times 10^{-30} \mathrm{~m}^{3}[2]$ and a typical line charge density $q=5 \times 10^{-8} \mathrm{C} / \mathrm{m}$, the coupling constant for angular momentum quantum number $m=1$ is found to be $\eta_{L i}^{2} \approx 0.78 \in[0,1)$. It is also possible to get other values of $\eta_{L i}$, such that it lies within the desired interval $\eta_{L i}^{2} \in[0,1)$ by increasing the line charge density $q$. Note that for $m=0$, the coupling constant $\eta_{L i}^{2}<0$ for any non-zero values of the line charge density $q$. In this case it is already known 22] (see section 6) that the spectrum is not bounded from below. Therefore the atom with $m=0$ will hit the nanotube and will be lost.

Let us now come back to our discussion. In our case we are dealing with a real symmetric (Hermitian) Hamiltonian $H_{r}$ over the domain

$$
\begin{aligned}
D\left(H_{r}\right) & \equiv \\
\{\phi(0) & \left.=\phi^{\prime}(0)=0, \phi, \phi^{\prime} \text { absolutely continuous }\right\}(3)
\end{aligned}
$$

Since the domain (3) is too restricted, it fails to be selfadjoint. Here we need to know that if the Hamiltonian is self-adjoint in a given domain $D$, then it should be equal to its adjoint Hamiltonian domain $D^{*}$, i.e., $D=D^{*}$. In our case the domain for our adjoint Hamiltonian $H_{r}^{*}$ is given by

$$
D\left(H_{r}^{*}\right) \equiv\left\{\phi \mid \phi, \phi^{\prime} \text { absolutely continuous }\right\} .
$$

It is clear from the above expression of $D\left(H_{r}\right)$ and $D\left(H_{r}^{*}\right)$ that $D\left(H_{r}\right) \neq D\left(H_{r}^{*}\right)$. So our Hamiltonian $H_{r}$ is not self-adjoint. For a non self-adjoint operator, there exists complex eigenvalue solutions for its adjoint operator, whereas for a self-adjoint operator there does not exist any complex eigenvalue solutions for its adjoint operator [20, 21]. So alternatively we can determine whether $H_{r}$ is self-adjoint in $D\left(H_{r}\right)$, by looking at the square integrable solutions of the equations

$$
H_{r}^{*} \phi_{ \pm}= \pm i \phi_{ \pm}
$$

The adjoint operator $H_{r}^{*}$ in our case is given by the same differential expression as $H_{r}$, but their domains are different. The square-integrable solutions for the + type and - type of (5) are called the deficiency space solutions and 
the number of solutions $n_{ \pm}$are called deficiency indices. According to the numbers $n_{+}$and $n_{-}$, the Hamiltonian $H_{r}$ can be divided into three classes:

Self-adjoint(essentially): for $\left(n_{+}, n_{-}\right)=(0,0)$.

Admit self-adjoint extensions: for $n_{+}=n_{-} \neq 0$.

No self-adjoint extensions possible: for $n_{+} \neq n_{-}$.

For the Hamiltonian $H_{r}$ we need to concentrate only on square-integrable solutions of (5) for our analysis. The two solutions which are square integrable at infinity are

$$
\phi_{+}(r)=r^{1 / 2} H_{\eta}^{(1)}\left(r e^{i \frac{\pi}{4}}\right), \phi_{-}(r)=r^{1 / 2} H_{\eta}^{(2)}\left(r e^{-i \frac{\pi}{4}}\right) .
$$

$H_{\eta}$ 's in (6) are Hankel functions [34]. We first consider the case $\eta \neq 0$. The short distance behavior $(r \rightarrow 0)$ of the functions $\phi_{ \pm}$are given by

$$
\begin{aligned}
& \phi_{+}(r) \simeq \mathcal{C}_{1}(\eta)\left(\frac{r}{2}\right)^{\eta+1 / 2}+\mathcal{C}_{2}(\eta)\left(\frac{r}{2}\right)^{-\eta+1 / 2} \\
& \phi_{-}(r) \simeq \mathcal{C}_{1}^{*}(\eta)\left(\frac{r}{2}\right)^{\eta+1 / 2}+\mathcal{C}_{2}^{*}(\eta)\left(\frac{r}{2}\right)^{-\eta+1 / 2}
\end{aligned}
$$

where $\mathcal{C}_{1}(\eta)=\frac{i}{\sin \eta \pi} \frac{e^{-i \frac{3 \eta \pi}{4}}}{\Gamma(1+\eta)}, \mathcal{C}_{2}(\eta)=-\frac{i}{\sin \eta \pi} \frac{e^{-i \frac{\eta \pi}{4}}}{\Gamma(1-\eta)}$ and $\mathcal{C}_{1}^{*}(\eta)$ and $\mathcal{C}_{2}^{*}(\eta)$ are complex conjugates of $\mathcal{C}_{1}(\eta)$ and $\mathcal{C}_{2}(\eta)$ respectively. The solutions $\phi_{ \pm}$are not square integrable near the singularity $(r \rightarrow 0)$ for $\eta^{2} \geq 1$. This means the deficiency indices are zero, i.e., $n_{+}=n_{-}=0$. So according to our classification scheme above, $H_{r}$ is essentially self-adjoint in the domain $D\left(H_{r}\right)$ [20, 21] for $\eta^{2} \geq 1$. But for $-1<\eta<0$ or $0<\eta<1$, the deficiency space solutions $\phi_{ \pm}$are square-integrable. Which means, $H_{r}$ has deficiency indices $(1,1)$ in this range and is not self-adjoint on the domain $D\left(H_{r}\right)$. Since the deficiency indices $n_{+}=n_{-}=1$ are equal, the Hamiltonian admits self-adjoint extensions. Since the case of zero deficiency indices i.e., $(0,0)$, indicates that the corresponding Hamiltonian is self-adjoint, any departure from $(0,0)$ shows the measure by which the Hamiltonian fails to be self-adjoint. In order to make it self-adjoint we then need to add the deficiency space solutions with the original domain and make it less restrictive such that the new domain becomes equal to its corresponding adjoint domain. In our present case also since the Hamiltonian $H_{r}$ is not self-adjoint in the domain $D\left(H_{r}\right)$, we need to add the deficiency space solutions $\phi_{ \pm}$to the domain $D\left(H_{r}\right)$ appropriately to make it less restrictive, such that the Hamiltonian in this new domain becomes self-adjoint. Thus the new domain $D_{\omega}\left(H_{r}\right)$ in which $H_{r}$ is self-adjoint is given by

$$
D_{\omega}\left(H_{r}\right) \equiv D\left(H_{r}\right)+\phi_{+}+\mathrm{e}^{i \omega} \phi_{-},
$$

where $\omega \in R(\bmod 2 \pi)[20,21]$. One can now evaluate the spectrum of the Hamiltonian $H_{r}$ taking the self-adjoint domain $D_{\omega}\left(H_{r}\right)$ into account.

One can evaluate the solution of the differential equation (2) as

$$
R(r) \equiv r^{1 / 2} H_{\eta}^{(1)}(\sqrt{\epsilon} r)
$$

The short distance $(r \rightarrow 0)$ behavior of $\phi_{+}(r)+e^{i \omega} \phi_{-}(r)$ is given by

$$
\begin{aligned}
& \phi_{+}(r)+e^{i \omega} \phi_{-}(r) \simeq \\
& \frac{2 e^{i \omega / 2}}{\Gamma(1+\eta)} \frac{\sin (\omega / 2+3 \pi \eta / 4)}{\sin \pi \eta}\left(\frac{r}{2}\right)^{\eta+1 / 2} \\
& -\frac{2 e^{i \omega / 2}}{\Gamma(1-\eta)} \frac{\sin (\omega / 2+\pi \eta / 4)}{\sin \pi \eta}\left(\frac{r}{2}\right)^{-\eta+1 / 2}
\end{aligned}
$$

The short distance behavior of (9) on the other hand is given by

$$
\begin{gathered}
R(r) \simeq \\
\mathcal{D}_{1}(\eta, \sqrt{\epsilon})\left(\frac{r}{2}\right)^{\eta+1 / 2}+\mathcal{D}_{2}(\eta, \sqrt{\epsilon})\left(\frac{r}{2}\right)^{-\eta+1 / 2}
\end{gathered}
$$

where $\mathcal{D}_{1}(\eta, \sqrt{\epsilon})=\frac{i}{\sin \pi \eta} \frac{e^{-i \pi \eta} \sqrt{\epsilon} \eta}{\Gamma(1+\eta)}$ and $\mathcal{D}_{2}(\eta, \sqrt{\epsilon})=$ $-\frac{i}{\sin \pi \eta} \frac{\sqrt{\epsilon}^{-\eta}}{\Gamma(1-\eta)}$. In order that $R(r)$ belongs to $D_{\omega}\left(H_{r}\right)$, the coefficients of $r^{\eta+1 / 2}$ and $r^{-\eta+1 / 2}$ in (10) and (11) should match. The bound state energy can be found by comparing the coefficients and the energy eigenvalue is given by

$$
E=-\frac{\hbar^{2}}{2 \mu}\left[\cos \frac{\pi \eta}{2}+\cot \left(\frac{\omega}{2}+\frac{\pi \eta}{4}\right) \sin \frac{\pi \eta}{2}\right]^{\frac{1}{\eta}}
$$

From (12), we can see that the system given by the Hamiltonian $H_{r}$ admits a single bound state solution. Note that not all values of $\omega$ allows a bound state. In fact only those values of $\omega$ admits a bound state for which the quantity in first bracket in (12) is positive. Since $\epsilon$ is negative, we can write down the the bound state eigenfunction (9) as

$$
R(r) \equiv r^{1 / 2} H_{\eta}^{(1)}(i \sqrt{|\epsilon|} r)
$$

The parameter $\omega$, in bound state eigenvalue and in the bound state eigenfunction indicates that each value of parameter $\omega$ characterizes one separate system, thus leading to inequivalent quantization.

One can also recalculate all the above things for $\eta=0$. The procedure is same. Here we only state the results for completeness. The bound state energy and the wave function for $\eta=0$ are given by

$$
\begin{aligned}
E & =-\frac{\hbar^{2}}{2 \mu} \exp \left[\frac{\pi}{2} \cot \frac{\omega}{2}\right], \\
R(r) & =\sqrt{-2 \epsilon r} K_{0}(\sqrt{-\epsilon} r),
\end{aligned}
$$

respectively, where $K_{0}$ is the modified Bessel function 34]. Note that the above analysis shows that for $-1<$ $\eta<1$, the radial Hamiltonian describing an atom in the electric field $E$ of a charged nanotube admits a single bound state. From the condition $-1<\eta<1$, we can easily check that the particle with arbitrarily small polarizability can form bound state in the electric field of charged nanotube provided the line charge is sufficient 


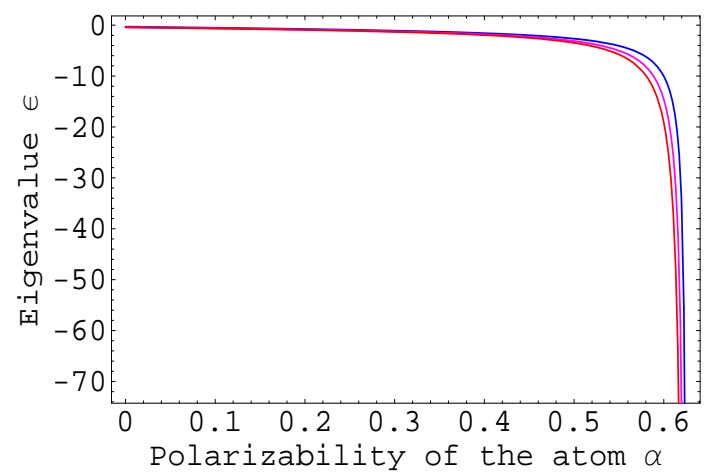

FIG. 2: (color online) A plot of binding energy of atom as a function of the polarizability $\alpha$ of the atom and with fixed line charge $q=1$ of the charged wire and for three different values of the self-adjoint extension parameter $\omega$. From top to bottom $\omega=\frac{\pi}{6}, \frac{\pi}{8}, \frac{\pi}{10}$ respectively.

to satisfy the condition $\eta^{2} \in[0,1)$. This conclusion is remarkably different from the statement in the literature that either the atom will fall into the charged nanotube or fly away depending upon the sign of the coupling constant $\eta^{2}$. Our model predicts the existence of a single bound state. The exact numerical value of the bound state energy would depend on the choice of the self-adjoint extension parameter $\omega$ which characterizes the boundary conditions at the origin. The bound state probability density for the atom has been plotted in FIG.1. It shows the nontrivial nature of the boundary condition, that the wave function is not zero at the origin, but still it is square integrable. In FIG. 2, bound state eigenvalue of the atom has been plotted as a function of the polarizability of the atom. It can be easily shown that the bound state eigenvalue of the atom as a function of the charge of the nanotube has the similar behavior.

We now address the important issue of smearing observed [1] in quantized conductance(QC). From our analysis of bound state formation we see that for $\eta^{2} \in[0,1)$, there is a single bound state, which depends on the selfadjoint extension parameter $\omega$. By suitably adjusting the parameter $\omega$, this bound state eigenvalue can be made large negative value. Now these deeply bound states will then contribute to the smearing of the edge of quantized conductance.

Let us now concentrate on breaking of scale invariance because of nontrivial quantization of the system. Since scale invariance is broken upon quantization, it naturally termed as quantum mechanical anomaly. Before discussing scale symmetry breaking let us discuss scale symmetry of this system by looking at its classical version. One can construct classical Lagrangian from the Hamiltonian (2) as $L=\frac{d^{2} r}{d t^{2}}-\frac{1}{r^{2}}\left(\eta^{2}-\frac{1}{4}\right)$. This lagrangian scales as $\frac{1}{\lambda^{2}} L$ under the scale transformation $r \rightarrow \lambda r$ and $t \rightarrow \lambda^{2} t$. One can also see that the action $\mathcal{A}=\int d t L$ for the system is invariant under the scale transformation. So the system is classically scale invariant. Scale invari- ance implies that if $\chi$ is a eigenstate of the Hamiltonian (2) with eigenvalue $-E, E>0$, then $\chi_{\lambda}=\chi(\lambda r)$ will also become an eigenstate of the same Hamiltonian (2) with eigenvalue $-E / \lambda^{2}$. By tuning $\lambda$ one can get any negative eigenvalue up to $-\infty$. It indicates that the system must not have any bound state. But we see that after quantization the system admits bound states for specific values of the parameter $\eta$. This violation of scale symmetry due to nontrivial quantization can be understood as follows. We first discuss the case for $\eta \neq 0$. In order to discuss scaling anomaly quantum mechanically we define a scaling operator $\Lambda=\frac{-i}{2}\left(r \frac{d}{d r}+\frac{d}{d r} r\right)$. It acts on any element $\phi$ belonging to the domain $D_{\omega}\left(H_{r}\right)$. In our case this scaling operator $\Lambda$ takes the wave-function $\phi$ out of the domain $D_{\omega}\left(H_{r}\right)$. It can be easily understood from the relation

$$
\begin{aligned}
& \lim _{r \rightarrow 0} \Lambda \phi(r) \simeq \\
& \quad \frac{(1+\eta)}{i} \frac{2 e^{i \omega / 2}}{\Gamma(1+\eta)} \frac{\sin (\omega / 2+3 \pi \eta / 4)}{\sin \pi \eta}\left(\frac{r}{2}\right)^{\eta+1 / 2} \\
& -\frac{(1-\eta)}{i} \frac{2 e^{i \omega / 2}}{\Gamma(1-\eta)} \frac{\sin (\omega / 2+\pi \eta / 4)}{\sin \pi \eta}\left(\frac{r}{2}\right)^{-\eta+1 / 2}
\end{aligned}
$$

Because of the two different multiplying factors $(1+\eta)$ and $(1-\eta)$ in (15), $\Lambda \phi(r) \neq C \phi(r)$ ( $C$ is any complex constant). So clearly $\Lambda \phi(r)$ does not belong to the domain $D_{\omega}\left(H_{r}\right)$. This shows that scale invariance is thus broken after inequivalent quantization of the system by self-adjoint extensions. However, scaling anomaly is not present for all values of the self-adjoint extension parameter $\omega$. For example, for $\omega=-\frac{\eta \pi}{2}$ and $\omega=-\frac{3 \eta \pi}{2}$, the action of the scaling operator $\Lambda$ on any function $\phi(r) \in D_{\omega}\left(H_{r}\right)$ does not throw it outside the domain. For these two values of the parameter $\omega$ the scaling symmetry is restored [17, 18, 19] and there does not exist any bound solution. We will not discuss $\eta=0$ case here, but one can discuss it similarly. For negative values of the parameter $\eta^{2}$, the usual analysis gives rise to bound states whose ground state goes to $-\infty$. It is however possible to use re-normalization group techniques 35]. But since it is in strong attractive region, it does not have so much importance as far as semaring is concerned.

In conclusion, here we shown that it is possible for polarizable neutral atom to form a single bound state in the electric field of the charged nanotube (SWNT). Nontrivial boundary conditions obtained by self-adjoint extensions makes it possible to get this bound state solution. We have used von Neumann method to make the initially non self-adjoint Hamiltonian $H_{r}$ self-adjoint. We have found out the domain $D_{\omega}\left(H_{r}\right)$ on which the Hamiltonian is self-adjoint. We have shown that in general the scaling operator $\Lambda$ acting on this domain $D_{\omega}\left(H_{r}\right)$ does not keep the domain invariant. But for two values of the selfadjoint extension parameter $\omega=-\frac{\eta \pi}{2}$ and $\omega=-\frac{3 \eta \pi}{2}$, scaling symmetry does not brake down. The existence of a bound state may contribute to the possible smearing of the edge of quantized conductance. The detection of atomic binding in the the electric field of the charged 
nanotube would be an interesting experiment. At least, within the experimental setup in Ref. [1] one should see the effect of bound state in scattering or absorption cross section.

\section{Acknowledgment}

We thank Kumar S. Gupta for encouraging us and helping us all the time throughout the preparation of the manuscript. The careful reading of the manuscript by $\mathrm{P}$. B. Pal is also acknowledged.
[1] T. Ristroph, A. Goodsell, J. A. Golovchenko and L. V. Hau, Phys. Rev. Lett. 94, 066102 (2005).

[2] J. Denschlag, G. Umshaus and J. Schmiedmayer, Phys. Rev. Lett. 81, 737 (1998)

[3] E. Arimondo, W. D. Philips and F. strumia (editors), Laser Manipulation of Atoms and Ions, Proceedings of the International School of Physics "Enrico Fermi" (North-Holland, Amsterdam, 1992).

[4] S. Chu, Rev. Mod. Phys. 70, 685 (1998).

[5] C. N. Cohen-Tannoudji, Rev. Mod. Phys. 70, 707 (1998).

[6] W. D. Phillips, Rev. Mod. Phys. 70, 721 (1998).

[7] L. V. Hau, M. M. Burns and J. A. Golovchenko, Phys. Rev A45, 6468 (1992).

[8] L. V. Hau, M. M. Burns and J. A. Golovchenko, Phys. Rev. Lett. 74, 3138 (1995).

[9] J. D. Weinstein and K. G. Libbrecht, Phys. Rev A52, 4004 (1995).

[10] J. Schmiedmayer, Appl. Phys. B60, 169 (1995).

[11] J. Denschlag and J. Schmiedmayer, Europhys. Lett. 38, 405 (1997).

[12] J. Gong, A. Ma and S. A. Rice, A72, 063410 (2005).

[13] L. D. Landau and E. M. Lifshitz, Quantum Mechanics, (Pergamon Press, London, 1959).

[14] K. M. Case, Phys. Rev. 80, 797 (1950).

[15] P. M. Morse and H. Feshbach, Methods of Theoretical Physics(McGraw-Hill, New York, 1953).

[16] V. M. Tkachuk, Phys. Rev. A60, 4715 (1999).

[17] B. Basu-Mallick, Pijush K. Ghosh and Kumar S. Gupta, Nucl. Phys. B659, 437 (2003).

[18] B. Basu-Mallick, Pijush K. Ghosh and Kumar S. Gupta, Phys. Lett. A311, 87 (2003).

[19] Kumar S. Gupta, Mod. Phys. Lett. A18, 2355 (2003).

[20] M. Reed and B. Simon, Fourier Analysis, Self-
Adjointness ( New York :Academic, 1975 ).

[21] N. Dunford and J. T. Schwartz, Linear Operators, Spectral Theory, Self Adjoint Operators in Hilbert Space, Part 2 (Wiley-Interscience; Wiley Clas edition, 1988).

[22] L. Feher, I. Tsutsui and T. Fulop, Nucl. Phys. B715, 713 (2005).

[23] H. Falomir, M. A. Muschietti and P. A. G. Pisani, J. Math. Phys. 45, 4560 (2004).

[24] H. Falomir, M. A. Muschietti, P. A. G. Pisani and R. Seeley, J. Phys. A36, 9991 (2003).

[25] H. Falomir, P. A. G. Pisani and A. Wipf, J. Phys. A35, 5427 (2002).

[26] B. Basu-Mallick and Kumar S. Gupta, Phys. Lett. A292, 36 (2001).

[27] D. Birmingham, Kumar S. Gupta and Siddhartha Sen, Phys. Lett. B505, 191 (2001).

[28] Kumar S. Gupta and Siddhartha Sen, Phys. Lett. B526, 121 (2002).

[29] S. Meljanac, A. Samsarov, B. Basu-Mallick and Kumar S. Gupta, Eur. Phys. J. C49, 875 (2007).

[30] H. E. Camblong, L. N. Epele, H. Fanchiotti and C. A. G. Canal, Phys. Rev. Lett. 87220402 (2001).

[31] H. E. Camblong, C. R. Ordonez, Phys. Rev. D68, 125013 (2003).

[32] P. R. Giri, K. S. Gupta, S. Meljanac and A. Samsarov, hep-th/0703121

[33] J. G. Esteve, Phys. Rev. D34, 674 (1986); Phys. Rev. D66, 125013 (2002).

[34] M. Abromowitz, I. A. Stegun, Handbook of Mathematical Functions (Dover, New York, 1970).

[35] K. S. Gupta and S. G. Rajeev, Phys. Rev. D48, 5940 (1993). 